\title{
Reinforcement of behavior with cues correlated with extinction'
}

RONALD E. SCHAUB ${ }^{2}$ AND WERNER K. HONIG

DALHOUSIE UNIVERSITY

Pigeons pecking at a white key produced both occasional primary reinforcement and brief red "cue" lights. This procedure altemated with periods of extinction during which pecking only produced green cues. After extensive training, pecking remained at a high rate during extinction components, suggesting that the green cue served as a reinforcer for pecking in extinction. Yoked Ss receiving the same cues non-contingently tended to cease responding in extinction.

It is frequently assumed that time-out from positive reinforcement is an aversive condition (Adelman \& Maatsch, 1956; Wagner, 1963). From this it follows that stimuli associated with extinction should acquire aversive properties, and should punish responses which produce them. This view has recently been questioned by Leitenberg (1965), who argues that the aversive nature of extinction periods depends greatly on the specific conditions of the experiment. The present study demonstrates that cues correlated with extinction may well have positively reinforcing properties.

In order to study this problem, it is necessary to make a concurrent assessment of the discriminative and reinforcing properties of the stimuli used in a discrimination procedure. This cannot be done in the typical discrimination study, where cues are generally presented for long periods and where their appearance is not contingent upon the S's behavior. In the present study, brief exteroceptive cues were presented to the Ss as discriminative stimuli (Holz \& Azrin, 1962) either contingent or not contingent upon their own behavior. When such cues are presented non-contingently, they can acquire a discriminative function. When they are made response-contingent, they can also serve a reinforcing function.

\section{EXPERIMENT 1}

\section{Method}

The first experiment was carried out with two pairs of domestic pigeons. They were first trained in two commercial operant behavior units to peck at a white illuminated key and given several sessions of 3-sec. mixed-grain reinforcements on a variable interval (VI) schedule with a mean inter-reinforcement interval of 1 min. In discrimination training, positive periods in which the food reinforcement was maintained alternated in the following sequence with negative periods in which no reinforcement was available: $8 \mathrm{~min}$. +; $4 \mathrm{~min}$. -; $4 \mathrm{~min}$. +; $8 \mathrm{~min}$-; $4 \mathrm{~min}$. +; $4 \mathrm{~min}$. -. The change between conditions occurred without any external signal. For the master bird in each pair, re- sponding changed the color of the white key for $1.5 \mathrm{sec}$. on a low fixed-ratio schedule (see below). During positive periods the color changed to red; during extinction, to green. For the yoked box, the key light changed in the same ways whenever such changes were produced by the master bird: thus, the only difference in the training procedures lay in the fact that the light changes were response-contingent for the master bird and non-contingent for the yoked birds. The programming of reinforcement during positive periods was independent of the occurrence of the cues, and the key light was turned off during the presentation of grain.

\section{Results}

The results are summarized in Fig. 1 in terms of the mean discrimination ratios for the master and yoked birds. This measure is obtained by dividing the responses emitted during the negative periods by the total number of responses during the entire session. With no evidence of discrimination, this ratio would be .50 ; as discrimination improves, the ratio approaches .00 . The difference between the mean discrimination ratios for master and yoked birds is also plotted, and the value of the fixed ratio on which the eues were produced is indicated between the two sets of curves.

Since the difference in discrimination ratios was generally positive, it is clear that the master birds responded more rapidly in the extinction periods than

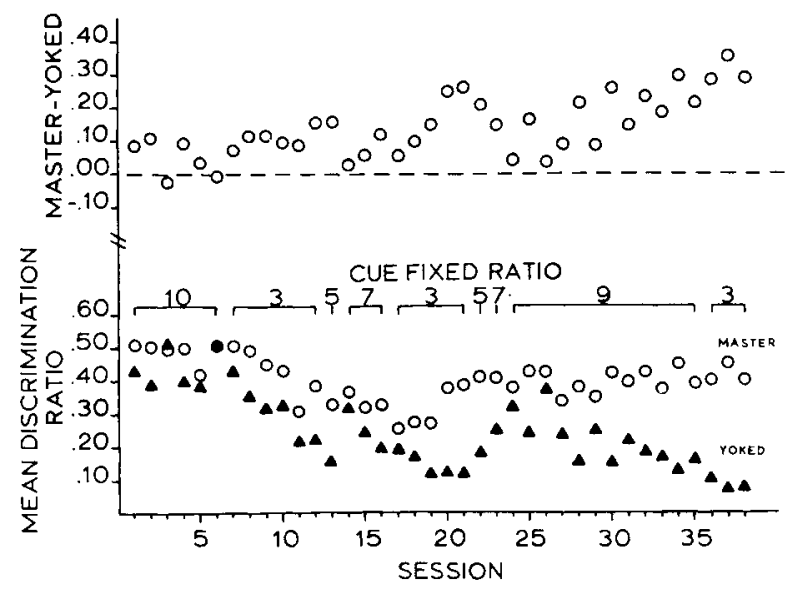

Fig. 1. Mean discrimination ratios and the difference between them obtained from yoked and master subjects in the first experiment. The number of responses required of the master birds to produce each cue at various stages of the experiment is indicated over the appropriate sessions as "cue fixed ratio." 


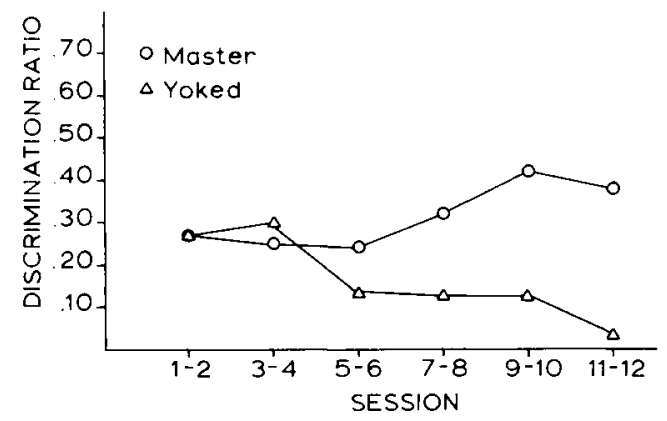

Fig. 2. Discrimination ratios obtained from one pair of $\mathrm{Ss}$ in the second experiment.

the yoked birds. This difference was small at the outset, when discriminative control was poor for the yoked Ss. It increased in the course of training, and tended to increase within blocks of sessions in which a given fixed ratio was in effect. In general, the difference was enhanced with small fixed ratios (FR 3) since this provided the yoked birds with frequent cues.

The pattern of responding was also quite different for master and yoked birds during negative periods. The yoked birds tended either to stop responding entirely, or to work at a rate similar to that emitted in positive periods. The master birds worked quite steadily through the entire negative periods, emitting a response pattern typical of low-value ratio schedules.

\section{EXPERIMENT 2}

A second experiment was designed so that the stimuli would acquire discriminative properties before they were produced by the animal's behavior. Two pairs of master and yoked birds first learned the discrimination in the presence of continuously presented red and green stimuli. The length of schedule components varied between 2 and $10 \mathrm{~min}$. When all birds were discriminating with a ratio of .05 or less, the white key illumination associated with original response acquisition was reinstated, and the response-cue contingency was put into effect on FR 3 in the manner described above. Results are presented in Fig. 2 for only one pair of birds since the yoked member of the second pair was removed from the study after six sessions due to illness. The ratio for both birds was close to .25 at the beginning of this procedure, reflecting an immediate loss of accuracy due to the shift from continuously to intermittently presented cues. The ratio for the master bird then increased while that for the yoked bird decreased. This demonstrates that the reinforcing function of the negative stimulus could counteract the previously acquired discriminative function and lead to the kind of increase in response rate which is one of the defining characteristics of a reinforcing stimulus.

\section{DISCUSSION}

From these results it appears that the production of a stimulus associated with extinction can be reinforcing, although the opposite would be expected if such cues are aversive. Since the positive cues may have become secondary reinforcers due to their temporal association with food reward, it is conceivable that the behavior of the master birds during the extinction periods was actually maintained by the production of the positive cues during reinforcement periods. While an explicit experimental test of this possibility has not been undertaken, this explanation is unlikely. It implies that the secondary reinforcing effects of the positive cues overrode the good discrimination that could be expected from the master birds on the basis of the performance of the yoked birds. We consider it more likely that the production of negative cues reinforced the behavior of the master birds directly. The critical factor in this reinforcement may have been the information provided by the discriminative stimuli on the reinforcement contingency. Such an effect would be closely related to the observing behavior recently reported for various segments of the inter-reinforcement interval in a fixed-interval schedule (Hendry \& Willow, 1966).

\section{References}

Adelman, H. M., \& Maatsch, J. L. Learning and extinction based upon frustration, food reward, and exploratory tendency. $J$. exp. Psychol., 1956, 52, 311-315.

Hendry, D. P., \& Willow, P. V. Observing behavior during interval schedules. J. exp. Anal. Behav., 1966, 9, 337-349.

Holz, W. C., \& Azrin, N. H. Interactions between the discriminative and aversive properties of punishment. J. exp. Anal. Behav., $1962,5,229-234$

Leitenberg, $H$. Is time-out from positive reinforcement an aversive event? Psychol. Bull., 1965, 64, 428-441.

Wagner, A. R. Conditioned frustration as a learned drive. J. exp. Psychol., 1963, 66, 142-148.

\section{Notes}

1. This research was supported by Grant APB-102 from the National Research Council of Canada to the second author.

2. Now at the Department of Psychology, State University of New York at Buffalo, Buffalo, N. Y. 14214. 